\title{
Ambient backscatter communication-based smart 5G loT network
}

\author{
Qiang Liu ${ }^{1 *}\left(\mathbb{B}\right.$, Songlin Sun ${ }^{1}$, Xueguang Yuan ${ }^{2}$ and Yang'an Zhang ${ }^{2}$
}

${ }^{*}$ Correspondence:
qiangliu@bupt.edu.cn
${ }^{1}$ School of Information
and Communication
Engineering, Beijing
University of Posts
and Telecommunications,
Beijing 100876, China
Full list of author information
is available at the end of the
article

*Correspondence: qiangliu@bupt.edu.cn 1 School of Information and Telecommunications, Full list of author information article

\begin{abstract}
In this paper, we propose an ambient backscatter communication-based smart 5G IoT network. The network consists of two parts, namely a real-time data transmission system based on ambient backscatter communication and a real-time big data analysis system based on the combination of shallow neural networks and deep neural networks. The real-time data transmission system based on ambient backscatter communication can extend the standby time of data collection equipment, reduce the size of the equipment, and increase the comfort of wearing. The real-time big data analysis system combining the shallow neural network and the deep neural network can greatly reduce the pressure caused by the frequent deep neural network calculations of the MEC and greatly reduce the energy consumed by the MEC for remote real-time monitoring.
\end{abstract}

Keywords: 5G, IOT, Machine learning, Ambient backscatter communication

\section{Introduction}

As the elderly population increases, the number of patients with chronic diseases will inevitably increase, which poses a huge challenge to the allocation of limited medical resources. The 5G Internet of Things (IoT) network based on artificial intelligence is widely regarded as a potential solution to alleviate the pressure on the healthcare system. 5G IoT networks need to consider the requirements for efficient use of spectrum, low latency, and high data rates. In order to meet the above requirements, it is necessary to combine artificial intelligence to make fast and effective decisions on the massive data generated by a large number of IoT devices [1]. With the development of cloud computing [2], MEC computing [3], artificial intelligence [4], IoT [5], network [6] based on big data analysis have been greatly developed. Smart 5G IoT networks use artificial intelligence as the driving force of big data analysis to analyze the collected health data, which can predict the possibility of chronic diseases, thereby reducing the workload of medical staff and reducing the pressure on the medical system.

Smart 5G IoT network based on big data analysis requires IoT devices or sensors to upload the collected massive data to the cloud or MEC for analysis. However, transferring data from IoT devices to the cloud or MEC is a huge challenge. If the IoT device performs wireless communication through active transmission, a certain amount of

(c) The Author(s) 2021. This article is licensed under a Creative Commons Attribution 4.0 International License, which permits use, sharing, adaptation, distribution and reproduction in any medium or format, as long as you give appropriate credit to the original author(s) and the source, provide a link to the Creative Commons licence, and indicate if changes were made. The images or other third party material in this article are included in the article's Creative Commons licence, unless indicated otherwise in a credit line to the material. If material is not included in the article's Creative Commons licence and your intended use is not permitted by statutory regulation or exceeds the permitted use, you will need to obtain permission directly from the copyright holder. To view a copy of this licence, visit http://creativecommons. org/licenses/by/4.0/. 
power is required. Limited battery capacity will limit the effective working time of IoT devices [7]. Increasing battery capacity will increase the standby time of IoT devices, but this will undoubtedly increase the volume of IoT devices. Large-capacity wearable IoT devices will unintentionally reduce wearing comfort [8]. Therefore, the volume and battery capacity of IoT devices will undoubtedly become one of the key factors for the widespread popularity of wearable devices. Therefore, transmission solutions for low-energy wearable IoT devices have become an important research direction in the development of IoT.

Backscatter communication is an emerging passive communication technology that can effectively solve the problems of sensor network communication and energy efficiency and is expected to become an effective data transmission solution for the network [9],[10]. Backscatter communications are mainly divided into three types, namely the monostatic backscatter communication system, bistatic backscatter communication system, and ambient backscatter communication system. Since ambient backscatter communication system is not equipped with any carrier transmitter, it is the most energy-efficient and lowest cost solution among the three backscatter communication solutions [11]. First, the ambient backscatter device use surrounding radio frequency (RF) sources for modulation without consuming additional energy. Secondly, by using the existing RF signal, it can work effectively without increasing the spectrum, thereby improving the spectrum efficiency. Finally, the low cost, small size, and low power consumption of ambient backscatter communication devices are conducive to the largescale deployment of low-power IoT devices. Especially in the field of health care, the ambient backscatter communication is a very promising solution for data transmission of implants and sensors in the body, which can effectively extend the running time of the device or sensor [12]. In fact, the potential benefits of ambient backscatter communication will greatly affect the data transmission method of networks.

In this paper, we propose a smart 5G IoT network based on ambient backscatter communication. The network consists of two parts, namely a real-time data transmission system based on ambient backscatter communication and a real-time big data analysis system based on the combination of shallow neural networks and deep neural networks.

The main innovation of the real-time data transmission system based on ambient backscatter communication is that we propose a cooperative ambient backscatter communication method. The cooperative ambient backscatter communication system transmits human vital signs data collected by in-body implants and on-body sensors, such as heart rate, blood pressure, body temperature, human $\mathrm{pH}$ and exercise status. Since the RF source will greatly affect the channel capacity of the backscatter system, the backscatter communication channel needs to be optimized to ensure the data transmission quality of in-body implants and on-body sensors. Without changing the transmission power of the BS, we proposed a novel backscatter coefficient adjust method. The method can maximize the backscatter transmission rate under a fixed power of the primary transmitter. This method analyzes the optimal value of the backscatter coefficient that cooperative receiver $(\mathrm{CR})$ can demodulate all the primary signals or only part primary signals. Then compare the optimal parameters in the two cases and make the parameter with the maximum backscatter transmission rate as the optimal backscatter coefficient of the ambient backscatter communication system. 
The real-time big data analysis system is based on the combination of shallow neural networks and deep neural networks, which can monitor and diagnose the physical condition of users. The system mainly includes three layers: data collection layer, MEC layer, and cloud layer. In the MEC layer, the collected data is first preprocessed, and then a shallow neural network is used to detect abnormalities in the preprocessed the physical condition data of users. If there is an abnormality, the abnormal data is further analyzed using a deep neural network, and finally the analysis results and health information are stored and managed in the cloud. The combination of shallow neural networks and deep neural networks can greatly reduce the pressure caused by frequent deep neural network calculations by MEC layer, and greatly reduce the energy consumed by MEC layer to perform remote real-time monitoring.

\section{System model}

The proposed smart 5G IoT network based on ambient backscatter communication is shown in Fig. 1. The network consists of two subsystems, the real-time data transmission system based on ambient backscatter communication, and the real-time big data analysis system based on the combination of shallow neural networks and deep neural networks. The real-time big data analysis system is the core of monitoring and diagnosing the physical condition of users, while the real-time data transmission system provides a powerful data transmission guarantee for the real-time big data analysis system. The real-time data transmission system is based on ambient backscatter communication, and a cooperative ambient backscatter communication method is proposed to ensure reliable data transmission. The real-time big data analysis system is based on the combination of shallow neural networks and deep neural networks, which can monitor and diagnose the physical condition of users.

\subsection{The real-time data transmission system}

The real-time data transmission system combines ambient backscatter communication and active communication, which can effectively guarantee data transmission and improve the stability and reliability of the system.

Active communication mainly includes communication technologies such as $\mathrm{Wi}-\mathrm{Fi}$, 4G, 5G [13-17], and wired networks. Active communication can transmit high-speed data transmission, such as videos and images. Its advantage is reliable and stable data transmission. Therefore, suitable active communication technologies can be selected according to different data transmission application scenarios. For example, high-speed active transmission can support remote high-definition video $(4 \mathrm{~K} / 8 \mathrm{~K})$ consultation and high-definition medical image data sharing and high-speed transmission. Besides, it enables experts to perform diagnosis and guidance anytime and anywhere, thereby extending high-quality medical resources to patients' homes. Active transmission technology enables a large number of video equipment and wearable medical equipment to capture and transmit patient health information in real time, thereby realizing real-time health information monitoring. In addition, in special wards such as infectious wards, medical staff can control active transmission-based medical assistant robots to move to designated beds to complete remote care services, thereby ensuring the safety of medical staff. 


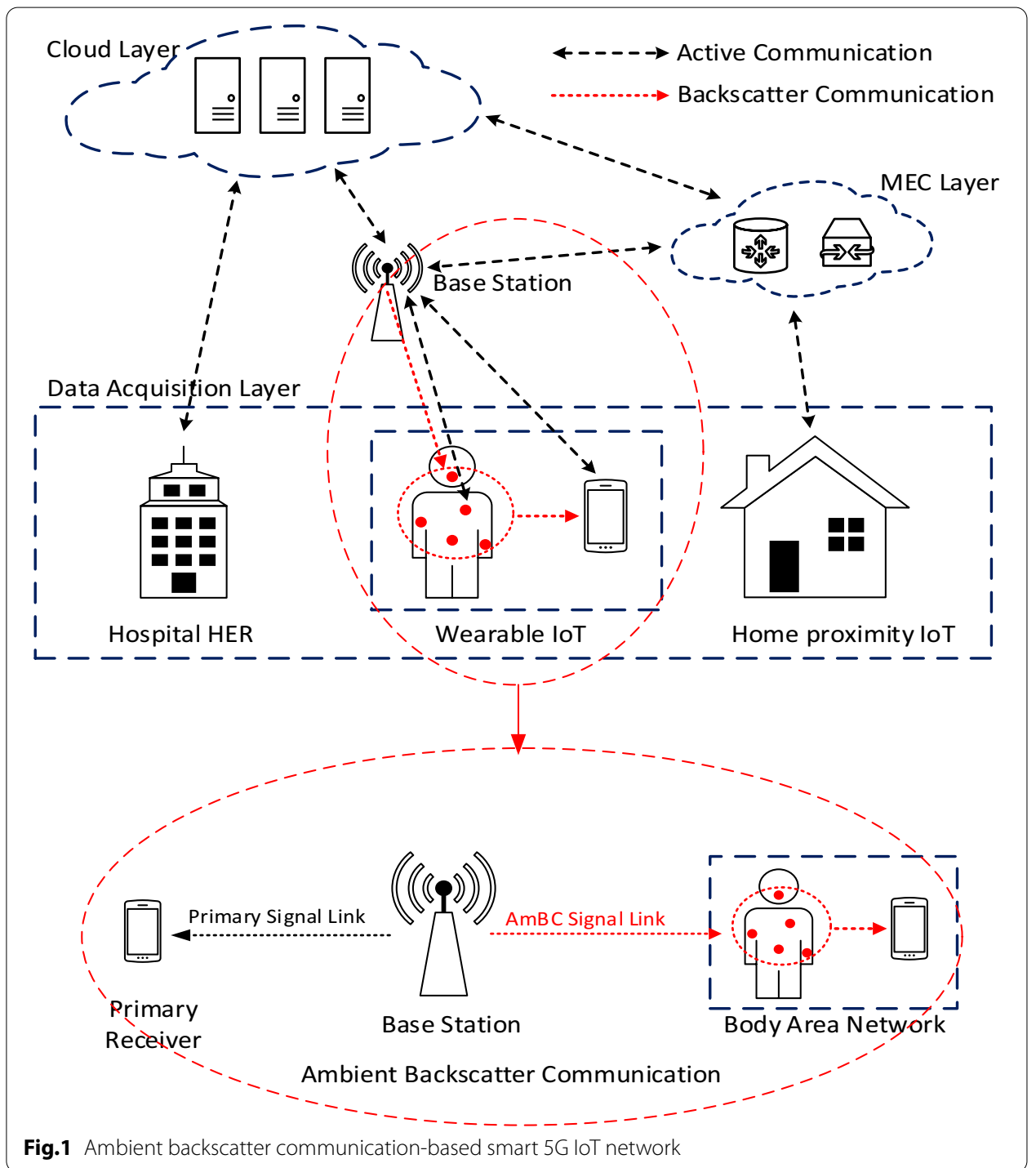

The ambient backscatter communication system is a very promising communication technology for low-cost IoT. In the ambient backscatter communication system, the passive IoT device can transmit data to the backscatter receiver by modulating and reflecting surrounding primary signals. The utilization of backscattering mechanism precludes the requirement of power-intensive RF-chain components like such as oscillators, analogto-digital converters, and power amplifiers, thereby greatly reduce the energy requirements of the circuit. When the distance between the receiver and the RF source does not change, as the distance between the RF source and the backscatter device increases, the received power of the receiver first decreases and then increases. The closer the backscatter device is to the receiver or RF source, the more power it receives. Therefore, as shown in Fig. 1, we use the user's handheld terminal such as a mobile phone as the receiver of the backscatter device, which is beneficial to increase the channel capacity of the backscatter device. The ambient backscatter communication system has the potential to increase the lifespan of wearable devices and human sensors without increasing 
the physical size of the device. Its low-power architecture can not only capture sensor data, but also transmit data within a few meters. By effectively exchanging data with a low power budget, it can completely change the way healthcare services are provided through embedded and wearable devices.

\subsection{The real-time big data healthcare analysis system}

The proposed framework of the real-time big data healthcare analysis system is shown in Fig. 2. The proposed framework consists of a data acquisition layer, a MEC layer and a cloud layer. The data collection layer consists of various IoT devices and sensors, which can effectively capture users' health-related data. The MEC layer uses shallow neural networks for anomaly detection and deep neural networks for in-depth analysis of detected anomalies. The cloud layer stores, manages and provides corresponding application services for the analysis results and health information from the MEC layer.

\subsubsection{Data acquisition layer}

The data collection layer provides data support for the medical big data analysis system. The data collection layer collects data from different data sources, including electronic hospital records (EHR), wearable IoT devices, proximity IoT devices and electronic questionnaires. EHR data usually includes patient visit records, medical diagnosis information and medication records. Wearable devices include on-body sensors and in-bodyimplanted devices, which can collect various health indicators of users in real time. Proximity IoT devices are placed in the user's daily life environment, with environmental information such as humidity, temperature, noise, air quality, and user's information such as the height and weight. Electronic questionnaires are used to regularly survey users' lifestyles: such as alcohol consumption frequency and smoking frequency.

\subsubsection{The MEC layer}

The MEC layer monitors and diagnoses the health of users based on the combination of shallow neural networks and deep neural networks. In the MEC layer, the collected data is preprocessed first, and then a shallow neural network is used to detect

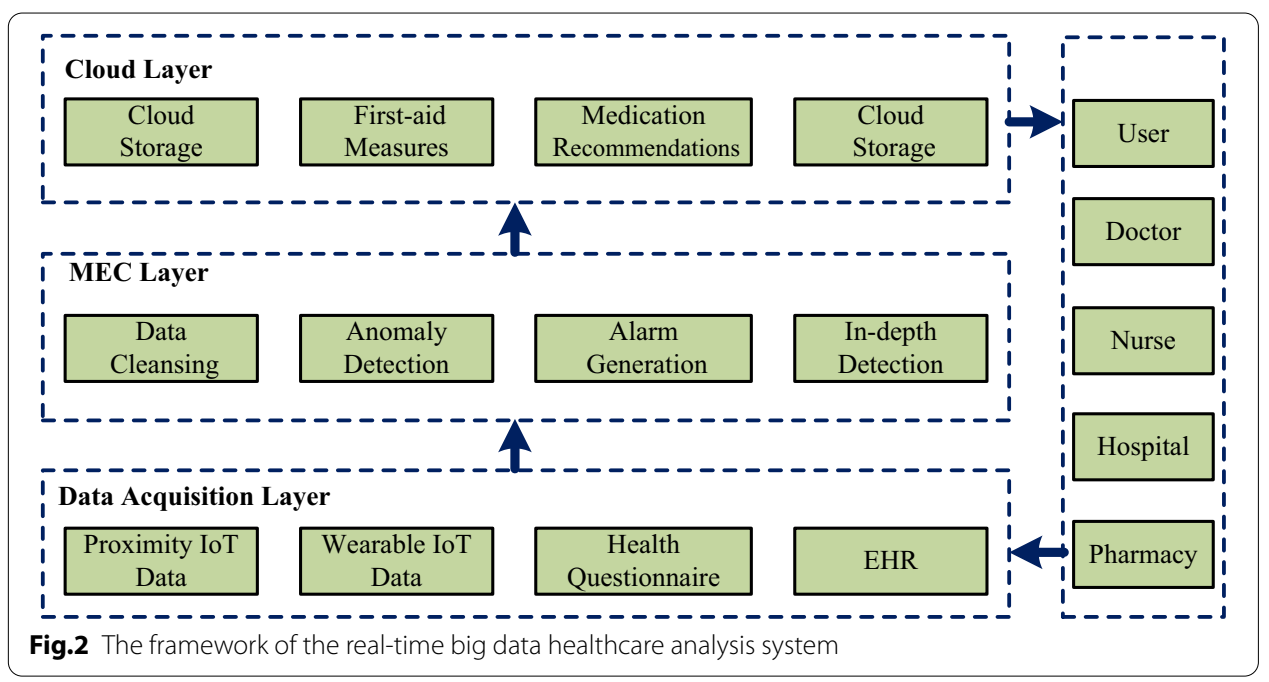


abnormalities in the preprocessed health data. If there is an abnormality, a deep neural network is used to further analyze the abnormal data, and finally the analysis results and health information are stored in the cloud and managed. The combination of shallow neural network and deep neural network can greatly reduce the pressure caused by frequent deep neural network calculations by MEC, and greatly reduce the energy consumed by MEC for remote real-time monitoring.

Data cleaning: The MEC layer collects data generated by the data acquisition layer. Due to fluctuations in the transmission link and data acquisition equipment, problems such as data redundancy or loss may occur. Therefore, it is necessary to replace incomplete or missing data with reasonable values and delete redundant data. Secondly, the data storage format of devices with the same functions from different manufacturers may be different. Therefore, it is necessary to convert these heterogeneous data into a unified format in the MEC layer for further analysis.

Anomaly detection: The process of judging whether a user is attacked by a certain disease is called anomaly detection. Anomaly detection is the prerequisite for realtime health monitoring at the MEC layer. The traditional anomaly detection mechanism is based on the threshold and does not consider the influence of other factors. Taking blood pressure (diastolic blood pressure (DBP), and systolic blood pressure (SBP)) as an example, the data obtained statically in a hospital or clinic may be completely different from the data obtained in actual scenes with different motion states. Therefore, only considering SBP and DBP without considering other factors to determine whether a user has cardiovascular disease will have a great hidden danger. For the above reasons, it is very necessary to use various health data collected in the MEC layer to comprehensively determine the user's health status. Since real-time detection requires frequent tracking of related health data, directly using deep neural networks to analyze the health of users will put tremendous pressure on the computing resources of the MEC layer. Therefore, in the anomaly detection stage, it is a good choice to only consider whether the user is attacked by a disease without considering the type of disease. Research shows that only detecting large categories without subdividing small categories can effectively reduce the computational complexity. Therefore, using a shallow neural network for anomaly detection is a good choice, which can effectively reduce the computational complexity.

Alarm generation: When anomaly detection detects that the user is attacked by a disease, an alarm message will be generated. Based on the alarm information, the MEC layer will collect more detailed data from the data collection layer for in-depth analysis. For example, when detecting abnormalities in cardiovascular diseases, if abnormalities are detected in the MEC layer, alarm information is generated. Based on the alarm information, the data collection layer needs to collect more detailed ECG information from the user to determine the type of the cardiovascular disease.

In-depth detection: in-depth detection based on deep neural networks is the core of the entire real-time health diagnosis. Based on the generated alarm information, Cloud Layer uses deep learning methods to analyze the user's disease types in detail. For example, [18] developed a deep neural network to classify and predict 10 Heart disease type in single-lead ECG signals and obtained better classification accuracy than cardiologists. 


\subsubsection{The Cloud layer}

Based on the analysis results of the MEC layer, the cloud layer will notify the user of the corresponding medication recommendations, preventive recommendations and necessary first aid measures based on different applications. Then, the cloud layer stores alarm information, user health-related data and analysis results, and edits each user's medical information to be shared among authorized medical personnel, users, pharmacies, hospitals, and medical professionals to better serve users provide services.

\section{The collaborative ambient backscatter communication scheme for real-time data transmission}

The proposed ambient backscatter communication system as shown in Fig. 3, the received signal at the IoT receiver is a mixed signal which consists of the backscatter signal from the backscatter device and the direct primary signal from the RF source. if the direct primary signal is treated as an interference signal at the IoT receiver, the backscatter transmission rate is extremely low. Therefore, suppressing the direct primary signal received by the $\mathrm{IoT}$ receiver is an effective way to improve the backscatter transmission rate. One effective solution is cooperative reception, where the IoT receiver can both decode the direct primary signal and the backscatter signal by using the successive interference cancellation (SIC) method. In [19], the authors maximize the backscatter transmission rate by optimizing the power of the primary transmitter (PT) and the reflection coefficient of BD. However, under a given power of the PT, the reflection coefficient range of $\mathrm{BD}$ that satisfy continuous interference cancellation conditions is not necessarily the optimal range. Although the reflection coefficient in this range can completely demodulate the direct primary signal, it also limits the power of the backscatter signal. We propose a novel backscatter coefficient adjustment method, which has a better backscatter transmission rate than the SIC method.

\subsection{Channel model}

We consider the ambient backscatter communication system, which consists of an active primary transmitter $(\mathrm{PT})$, a primary receiver (PR), a passive backscatter device (BD), and

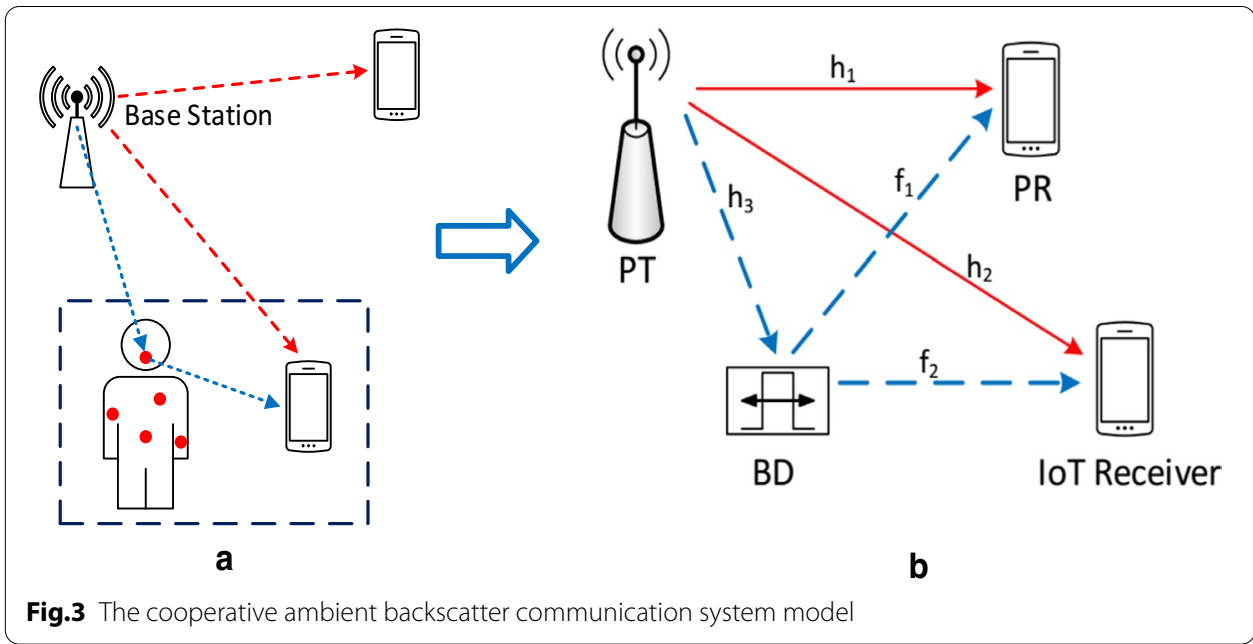


an IoT receiver as shown in Fig. 4. The IoT receiver is a cooperative receiver (CR), which can demodulate all or part of the primary signals via SIC technique. In this paper, all the relevant channels are assumed as block fading channels, where all the channel state information (CSI) unchanged in the same block. The CSI is assumed perfect, which can be estimated by channel estimation techniques. Then the channel gain for the block $n$ of PT-PR channel, PT-CR channel, PT-BD channel, BD-CR channel, and BD-PR channel can be denoted as $h_{1}(n), h_{2}(n), h_{3}(n), f_{1}(n), f_{2}(n)$, respectively.

\subsection{Transmission model}

\subsubsection{Transmitted signal at PT}

Let $s(n, k)$ denote the primary information signal transmitted from PT to PR at the kth symbol in the nth block, where $|s(n, k)|^{2}=1$. And let $p(n)$ denote the transmit power for the primary receiver in the nth block, where the $p(n)$ is assumed fixed in this letter. Then the transmitted signal at PT to the primary receiver for the kth symbol in the $n$th block is given by

$$
x_{P T}(n, k)=\sqrt{p(n)} * s(n, k) .
$$

\subsubsection{Transmitted signal at $B D$}

The received signal at $\mathrm{BD}$ from $\mathrm{PT}$ is $\sqrt{h_{3}(n)} * x_{P D}(n, k)$. Since the $\mathrm{BD}$ is a passive device, which does not consist any active FR component, the noise at BD can be neglected. let $c(n, k)$ denote the backscatter information signal transmitted from $\mathrm{BD}$ to $\mathrm{CR}$ at the nth symbol in the kth block, where $E\left(|c(n, k)|^{2}\right)=1$. Then the transmit signal at $\mathrm{BD}$ is given by

$$
x_{B D}(n, k)=\sqrt{h_{3}(n)} * \sqrt{\alpha} * x_{P T}(n, k) * c(n, k),
$$

where $\alpha$ denotes the reflection coefficient, which is the ratio of reflected signal power to incident signal power, where $0 \leq \alpha \leq 1$.

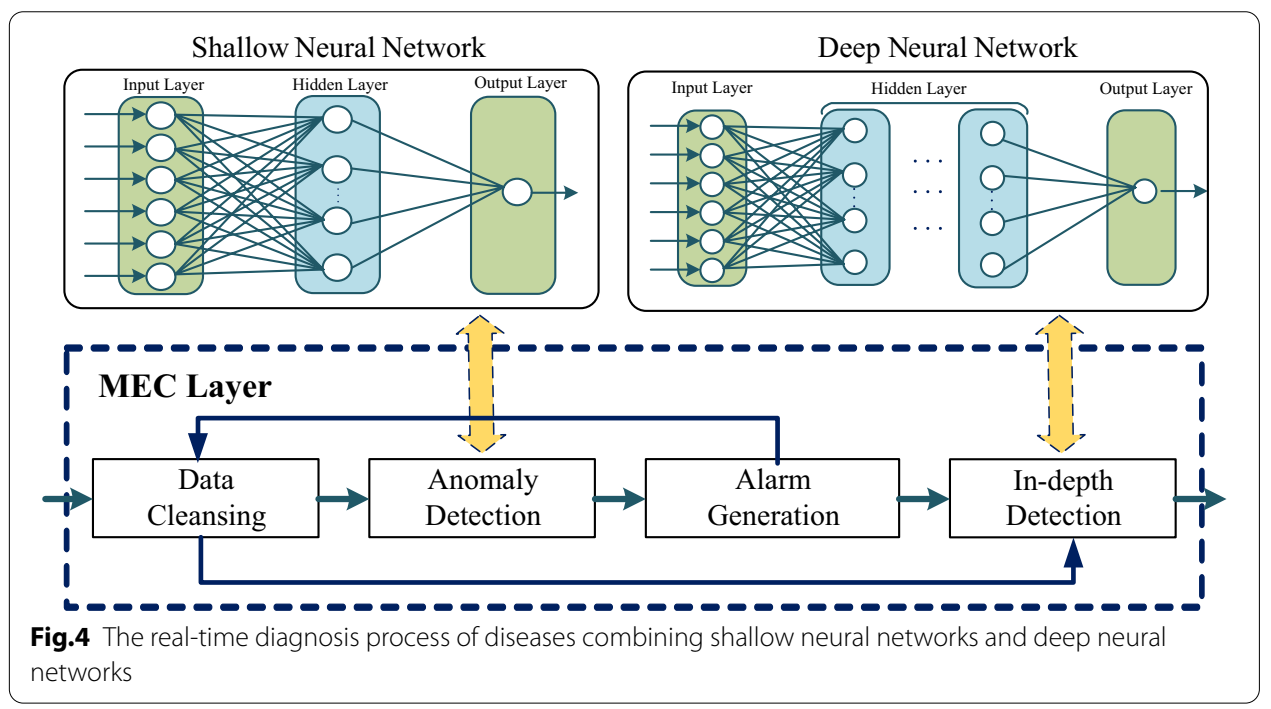




\subsubsection{Received signal at $P R$}

Let $y_{P R}(n, k)$ denote the received signal at the PR for the kth symbol in the $n$th block, then the received signal is given by

$$
y_{P R}(n, k)=\sqrt{h_{1}(n)} * x_{P T}(n, k)+\sqrt{f_{1}(n)} * x_{B D}(n, k)+n_{P R}(n, k),
$$

where $n_{P R}(n, k) \sim C N(0,1)$ denotes the normalized additive noise received at RP. The second component of (3) is the interference signal from BD, which can't be ignored. In addition, the delay between the PT-PR channel and the PT-BD-PR channel is assumed to be zero, which is widely accepted in the backscatter communication research. Then the instantaneous signal to interference plus noise ratio (SINR) for decoding the primary information signal is given by.

$$
\operatorname{SINR}_{P R}=\frac{P h_{1}(n)}{P \alpha h_{3}(n) f_{1}(n)+1}
$$

and the corresponding achievable transmission rate of primary information signal from $\mathrm{PT}$ to $\mathrm{PR}$ is given by.

$$
R_{P R}^{S}=\log _{2}\left(1+\operatorname{SINR} R_{P R}\right)
$$

\subsubsection{Received signal at $C R$}

Let $y_{C R}(n, k)$ denote the received signal at the CR for the kth symbol in the nth block, then the received signal at $C R$ is given by

$$
y_{C R}(n, k)=\sqrt{h_{2}(n)} * x_{P T}(n, k)+\sqrt{f_{2}(n)} * x_{B D}(n, k)+n_{C R}(n, k),
$$

where $n_{C R}(n, k) \sim \mathrm{CN}(0,1)$ denotes the normalized additive noise received at $\mathrm{CR}$. In this paper, we assume that the $C R$ decades the received signal by performing successive interference cancellation (SIC). Ideally, we can decode $x_{P T}(n, k)$ via SIC in $y_{C R}(n, k)$, thus $\sqrt{h_{2}(n)} * x_{P T}(n, k)$ can be eliminated from $y_{C R}(n, k)$. The SINR for decoding the primary information signal at $\mathrm{CR}$ is given by

$$
\operatorname{SINR}_{P R}^{s}=\frac{p h_{2}(n)}{\alpha p h_{3}(n) f_{2}(n)+1}
$$

and the corresponding achievable transmission rate of the primary information signal from $\mathrm{PT}$ to $\mathrm{BD}$ is given by.

$$
R_{C R}^{S}=\log _{2}\left(1+\operatorname{SINR}_{P R}^{S}\right) .
$$

If the $x_{P T}(n, k)$ can be decoded, the $x_{P T}(n, k)$ can be eliminated in $(6)$. Then the SINR for decoding the backscatter information signal is given by

$$
\operatorname{SINR}_{C R}^{c 1}=\alpha P h_{3}(n) f_{2}(n),
$$

and the corresponding achievable transmission rate of the backscatter information signal received at $C R$ is given by. 


$$
R_{C R}^{c 1}=\log _{2}\left(1+\operatorname{SINR}_{P R}^{c 1}\right) .
$$

If the $x_{P T}(n, k)$ cannot be decoded, $x_{P T}(n, k)$ needs to be treated as interference. In this case, the SINR for decoding the backscatter information signal at CR is given by.

$$
S N R_{C R}^{c 2}=\frac{\alpha P h_{3}(n) f_{2}(n)}{p h_{2}(n)+1},
$$

and the corresponding achievable data rate of the backscatter information signal received at $\mathrm{CR}$ is given by.

$$
R_{C R}^{c 2}=\log _{2}\left(1+\operatorname{SINR}_{C R}^{c 2}\right)
$$

\subsection{Transmission analysis}

Our objective is to maximize the achievable transmission rate of the backscatter information signal at $\mathrm{BD}$, while guaranteeing the minimum achievable transmission rate of the primary information signal at PR based on the QoS requirement. Denoted the minimum achievable transmission rate requirement at $\mathrm{PR}$ as $R_{P R}^{Q}$.

To guarantee the QoS requirement of PR, the minimum achievable transmission rate of the primary information signal at the PR should satisfy the following constraint

$$
R_{P R}^{S} \geq R_{P R}^{Q}
$$

Since the BD is a passive device, the signal after reflection must be less than or equal to the signal before reflection. Thus, the reflection coefficient constraint is given by

$$
0 \leq \alpha \leq 1 .
$$

When $R_{P R}^{s} \geq R_{P R}^{Q}$, the $C R$ can decode all the primary information signal. Then the achievable transmission rate of the backscatter information signal at $C R$ is equal to $R^{c 1}{ }_{C R}$. However, when $R_{P R}^{s}<R_{P R}^{Q}$, the CR can decode part of the primary information signal. Then the achievable transmission rate of the backscatter information signal at $\mathrm{CR}$ is equal to $R_{C R}^{c 1}$, where $\eta$ is the ratio of $R_{P R}^{s}$ to $R_{P R}^{Q}$. Denote the achievable transmission rate of the backscatter information signal at CR as $Q(\alpha)$ ), then $Q(\alpha)$ is given by

$$
Q(\alpha)=\left\{\begin{array}{cl}
R_{C R}^{c 1} & R_{C R}^{s} \geq R_{P R}^{Q} \\
\eta R_{C R}^{c 1}+(1-\eta) R_{C R}^{c 2} & R_{C R}^{s}<R_{P R}^{Q}
\end{array}\right.
$$

\subsection{Optimal reflection coefficient adjustment}

In this subsection, we adjust the optimal $\alpha$ to maximize $\mathrm{Q}(\alpha)$. This optimization problem can be represented as follows:

$$
\begin{aligned}
& \max _{\alpha} \mathrm{Q}(\alpha) \\
& \text { s.t. } R_{P R}^{s} \geq R_{C R}^{Q}
\end{aligned}
$$




$$
0 \leq \alpha \leq 1
$$

which is a non-convex optimization problem. We cannot solve (16a) by the standard convex optimization techniques. To solve this problem, we need to discuss $R_{P R}^{s} \geq R_{C R}^{Q}$ and $R_{P R}^{s}<R_{C R}^{Q}$, separately.

When $R_{P R}^{S} \geq R_{C R}^{Q}$, then (16) can be rewritten as follows

$$
\begin{aligned}
& \max _{\alpha} Q_{1}(\alpha)=R_{C R}^{c 1} \\
& \text { s.t. } R_{P R}^{s} \geq R_{P R}^{Q} \\
& R_{C R}^{S} \geq R_{P R}^{Q} \\
& 0 \leq \alpha \leq 1
\end{aligned}
$$

The first-order derivative of $\operatorname{SINR}_{C R}^{c 1}$ respect to $\alpha$ are given by

$$
\operatorname{SINR}_{C R}^{c 1}{ }^{\prime}=\frac{p h_{3}(n) f_{2}(n)}{1+\alpha p h_{3}(n) f_{2}(n)} .
$$

Then the first-order derivative of $R_{C R}^{c 1}$ with respect to $\alpha$ are given by

$$
R_{C R}^{c 1}{ }^{\prime}=\frac{\operatorname{SINR}_{C R}^{c 1}{ }^{\prime}}{\operatorname{SINR}_{C R}^{c 1}+1} .
$$

Due to $h_{1}(n)>0, h_{2}(n)>0, h_{3}(n)>0, f_{1}(n)>0, f_{2}(n)>0, p>0, \alpha>0$ we can get $R_{C R}^{c 1}{ }^{\prime}>0 . R_{C R}^{c 1}$ is a monotonically increasing function of $\alpha$.

Since SINRc1CR is an increasing function, we only need to find the maximum value of $\alpha$ that satisfies the constraint (17b), (17c), (17d). Then the value of $\alpha$ that satisfies the constraint (17b) is given by

$$
\alpha_{1}{ }^{(1)} \leq \frac{p h_{1}(n)+1-2^{R_{P R}^{Q}}}{\left(2^{R_{P R}^{Q}}-1\right) p h_{3}(n) f_{1}(n)} .
$$

The maximum value of $\alpha$ that satisfies the constraint (17c) is given by

$$
\alpha_{1}{ }^{(2)} \leq \frac{p h_{2}(n)+1-2^{R_{P R}^{Q}}}{\left(2^{R_{P R}^{Q}}-1\right) p h_{3}(n) f_{2}(n)} .
$$

Then the optimal solution of the reflection coefficient to the problem (16) is given by

$$
\alpha_{1}=\min \left(\alpha_{1}{ }^{(1)}, \alpha_{1}{ }^{(2)}, 1\right) .
$$

When $R_{P R}^{s}<R_{C R}^{Q}$, then (16) can be rewritten as follows

$$
\max _{\alpha} Q_{2}(\alpha)=\eta R^{c 1}{ }_{C R}(n)+(1-\eta) R_{C R}^{c 2}(n)
$$




$$
\begin{aligned}
& \text { s.t.R } \\
& P R \\
& R_{P R}^{S}<R_{P R}^{Q} \\
& \\
& 0 \leq \alpha \leq 1
\end{aligned}
$$

The value of $\alpha$ that satisfies the constraint (23b) is given by

$$
\alpha_{2}{ }^{(1)} \leq \frac{p h_{2}(n)+1-2^{R_{P R}^{Q}}}{\left(2^{R_{P R}^{Q}}-1\right) p h_{3}(n) f_{2}(n)} .
$$

The value of $\alpha$ that satisfies the constraint (23c) is given by

$$
\alpha_{2}{ }^{(2)} \leq \frac{p h_{2}(n)+1-2^{R_{P R}^{Q}}}{\left(2^{R_{P R}^{Q}}-1\right) p h_{3}(n) f_{1}(n)} .
$$

According to (23d), (24) and (25), the range of reflection coefficient of problem (23) is given by

$$
\max \left(\alpha_{2}{ }^{(2)}, 0\right) \leq \alpha \leq \min \left(\alpha_{2}{ }^{(1)}, 1\right)
$$

Then problem (21) can be reformulated as

$$
\begin{aligned}
& \max _{\alpha} Q_{2}(\alpha)=\eta R_{C R}^{c 1}+(1-\eta) R_{C R}^{c 2} \\
& \text { s.t. } \max \left(\alpha_{2}^{(2)}, 0\right) \leq \alpha \leq \min \left(\alpha_{2}^{(1)}, 1\right)
\end{aligned}
$$

Then, the first- and second-order derivative of $Q_{2}(\alpha)$ are given by

$$
\begin{aligned}
& Q_{2}^{\prime}(\alpha)=\eta^{\prime} R_{C R}^{c 1}-\eta^{\prime} R_{C R}^{c 2}+\eta R_{C R}^{c 1}{ }^{\prime}+(1-\eta) R_{C R}^{c 2}{ }^{\prime}, \\
& Q_{2}^{\prime \prime}(\alpha)=\eta^{\prime \prime} R_{C R}^{c 1}-\eta^{\prime \prime} R_{C R}^{c 2}+\eta^{\prime} R_{C R}^{c 1}{ }^{\prime}-\eta^{\prime} R_{C R}^{c 2}{ }^{\prime}+\eta^{\prime} R_{C R}^{c 1}{ }^{\prime}-\eta^{\prime} R_{C R}^{c 2}{ }^{\prime}+\eta R_{C R}^{c 1}{ }^{\prime \prime}+(1-\eta) R_{C R}^{c 2}{ }^{\prime} .
\end{aligned}
$$

However, for the complexity of $Q_{2}^{\prime}(\alpha)$ and $Q_{2}^{\prime \prime}(\alpha)$, it is difficult to judge the convexity of $Q_{2}(\alpha)$ and find the zero of $Q_{2}^{\prime}(\alpha)$. Therefore, we use the line search method to approximate the optimal value of reflection coefficient. Denote the value of reflection coefficient search by the line search method as $\alpha_{2}$. Then the optimal reflection coefficient is given by

$$
\alpha_{\text {optical }}=\left\{\begin{array}{ll}
\alpha_{1} & Q_{1}\left(\alpha_{1}\right) \geq Q_{2}\left(\alpha_{2}\right) \\
\alpha_{2} & Q_{1}\left(\alpha_{1}\right)<Q_{2}\left(\alpha_{2}\right)
\end{array} .\right.
$$




\section{Hybrid neural network architecture design for real-time big data healthcare analysis}

With the continuous development of medical technology, cloud computing based IoT medical systems have become one of the potential research directions. However, with the continuous development of IoT medical systems, cloud computing is facing more and more challenges. As the number of users continues to increase, a large amount of medical data needs to be uploaded to the cloud, which will put tremendous pressure on the core network and cloud layer. MEC computing can provide users with faster computing services, thereby making real-time analysis and decision-making more effective and feasible. In addition, MEC computing can also provide computing, storage and control services for IoT medical systems to meet various medical needs.

Taking these facts into account, we combine a shallow neural network and a deep neural network in the MEC layer to monitor and diagnose the health of users. The advantage of a shallow neural network is that it has only a few limited inputs and few hidden layers, which can quickly analyze anomalies and generate analysis results. However, due to limited input data and simple hidden layers, it is impossible to analyze specific disease types. Therefore, analyzing the needs of specific disease types requires more specific data and a more targeted neural network structure. Although deep neural networks can diagnose disease types, they are too complex and computationally intensive. When hundreds of patients need to be diagnosed at the same time, the MEC layer will be under tremendous pressure. Therefore, it is a very effective solution to combine the shallow neural network and the deep neural network in the MEC layer to monitor and diagnose the health of users.

The real-time diagnosis process of diseases combining the shallow neural network and the deep neural network is shown in Fig. 4. In the MEC layer, the collected data is first preprocessed, and the shallow neural network is used to determine whether the user's health status is abnormal. If there is an abnormality, the deep neural network is used to further analyze the abnormal data, and finally the analysis results and health information are stored in the cloud and managed. The combination of shallow neural network and deep neural network can greatly reduce the pressure caused by frequent deep neural network calculations by MEC, and greatly reduce the energy consumption of MEC for remote real-time monitoring.

In this article, we mainly predict the attack of heart disease. Since heart disease is a type of cardiovascular disease, we can first determine whether there is a risk of cardiovascular disease outbreak in the MEC layer. If users are at risk, we will collect more detailed ECG information for in-depth analysis and determine whether users will be at risk of a heart attack.

\subsection{Shallow neural network for anomaly detection}

The causes of cardiovascular disease are complex: for example, anxiety, stress, exercise, and drinking can cause sudden death in patients with cardiovascular disease. Therefore, it is necessary to design a real-time monitoring system to determine the possibility of cardiovascular disease so that patients can take emergency measures to avoid sudden death. The shallow neural network used in the MEC layer can determine 
the risk status of cardiovascular disease by considering attributes related to cardiovascular disease.

The proposed shallow artificial neural networks (ANN) is shown in Fig. 5. The input layer has 11 units, and the hidden layer has 16 neurons and 1 output node. There are three main types of input data: (1) The user's physical biochemical characteristics include glucose level, cholesterol level, SBP, DBP; (2) User habits include physical activity, alcohol intake, and smoking; (3) User's basic information includes gender, height, weight and age.

When the shallow neural network detects that the user is at risk of cardiovascular disease, it will generate an abnormal alert. Based on abnormal alarms, the data collection layer will collect relevant data for the next in-depth analysis. In this paper, we focus on whether the user has a heart attack, so the data collection layer will collect the user's current ECG data for the next in-depth examination.

\subsection{Deep neural network for in-depth detection}

When the shallow neural network detects that the user is at risk of cardiovascular disease, MEC will collect the user's current ECG information for in-depth analysis. As shown in Fig. 6, we trained a 22-layer convolutional neural network (CNN) to detect the type of heart disease in the ECG time series. In order to make the optimization of the convolutional neural network easy to handle, we use techniques such as residual connection and batch normalization. The residual block is composed of batch normalization, dropout, activation function and convolution function, these functions are connected sequentially and looped 3 times. At the same time, a Max Pooling layer is placed after the residual block structure to reduce the number of model parameters and the overfitting effect of the model. The last two full connect layers are used to output four classification results, which are normal, premature beats, heart block and interference.

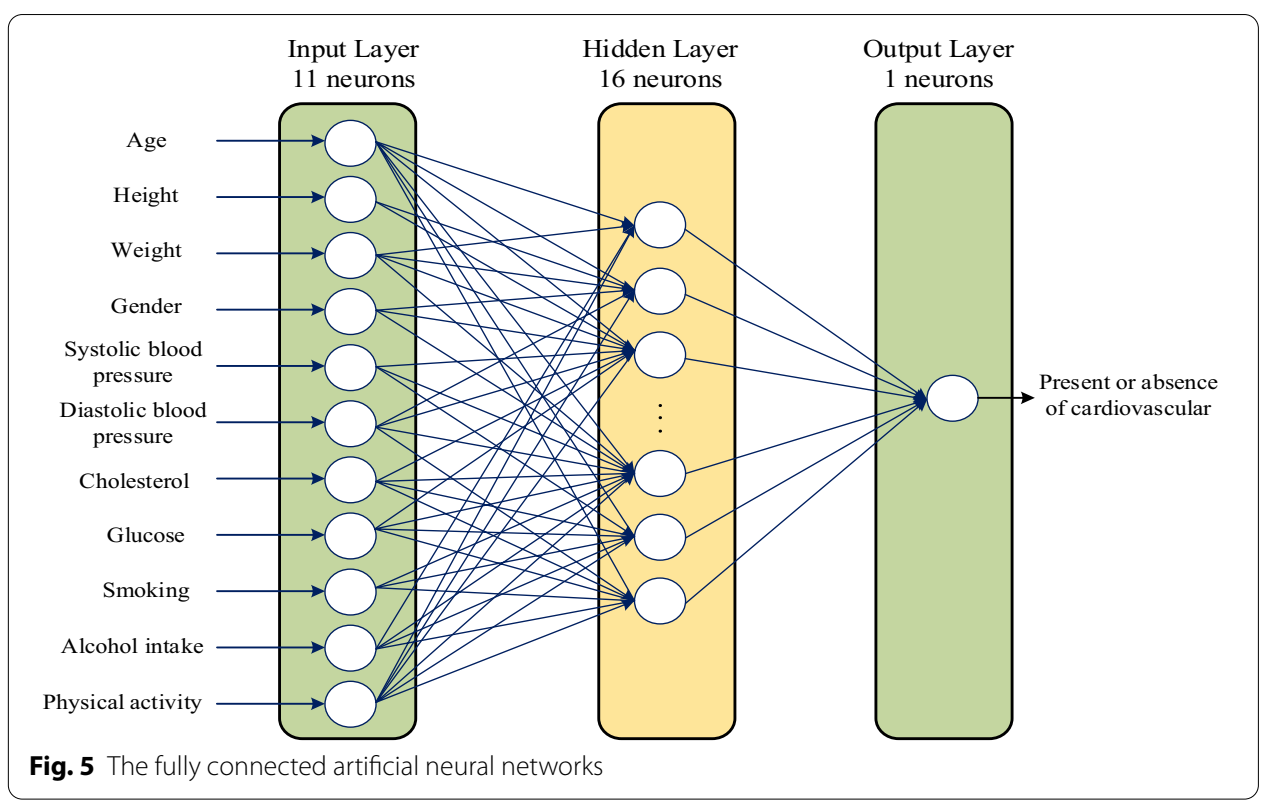




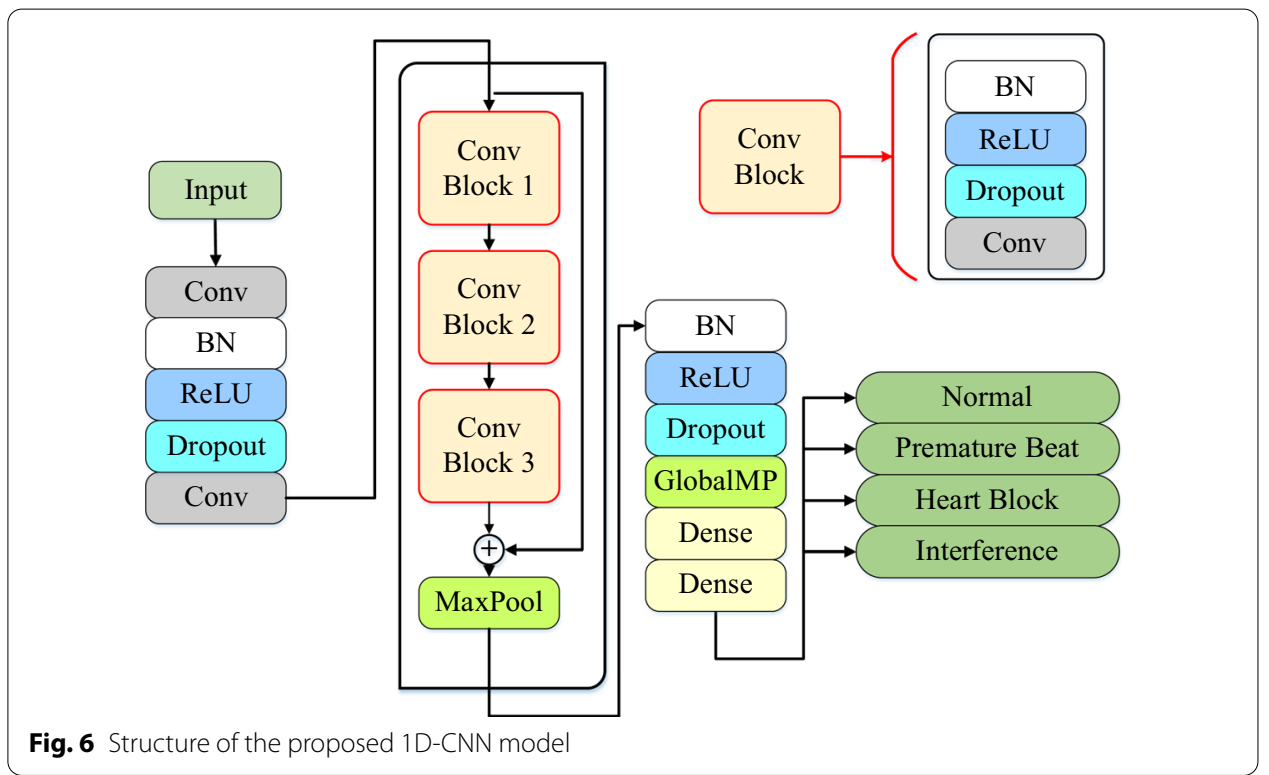

The proposed convolutional neural network will learn to classify the types of heart disease existing in the time series. The output of the proposed convolutional neural network is divided into four types: normal, premature beat, heart block and interference. The interference in these four classification types is defined as waveform distortion caused by transmission errors or excessive body motion during data acquisition. Although interference does not belong to any type of heart disease, it makes sense to include interference in the classification output due to the instability of data collected by wearable devices.

\section{Experimental simulation results and analysis}

In this section, we use two data sets to verify the effectiveness of the system. One data set is used for heart disease abnormality detection, and the other data set is used for heart disease diagnosis and analysis. Next, we will introduce these two data sets in detail.

We use the cardiovascular disease set published by Kaggle as the data set for the MEC layer abnormality detection based on shallow neural networks. The data set contains 70,000 patient data records, which contain 11 health-related features, such as gender, height, weight, age, physical activity, alcohol intake, smoking, glucose level, cholesterol level, SBP, DBP, and a disease label, i.e. presence or absence of cardiovascular disease. We use $80 \%$ of the data as the training set and $20 \%$ of the data as the test set.

To train and test the proposed deep learning model, the ECG data set used in this study contains a total of 102,899 records. A total of four categories of data sets are included. Normal has 66,384 data, the premature beat has 11,065 data, block heart has 5722 data and Interference has 19,728 data. It can be seen that the normal category accounts for a large part of the data set, while the number of other categories is relatively small, the least of which is premature beats, which only accounts for $6 \%$ of all records in the training set. Similarly, we divide the data set into two parts, $80 \%$ as the training set and $20 \%$ as the test set. 

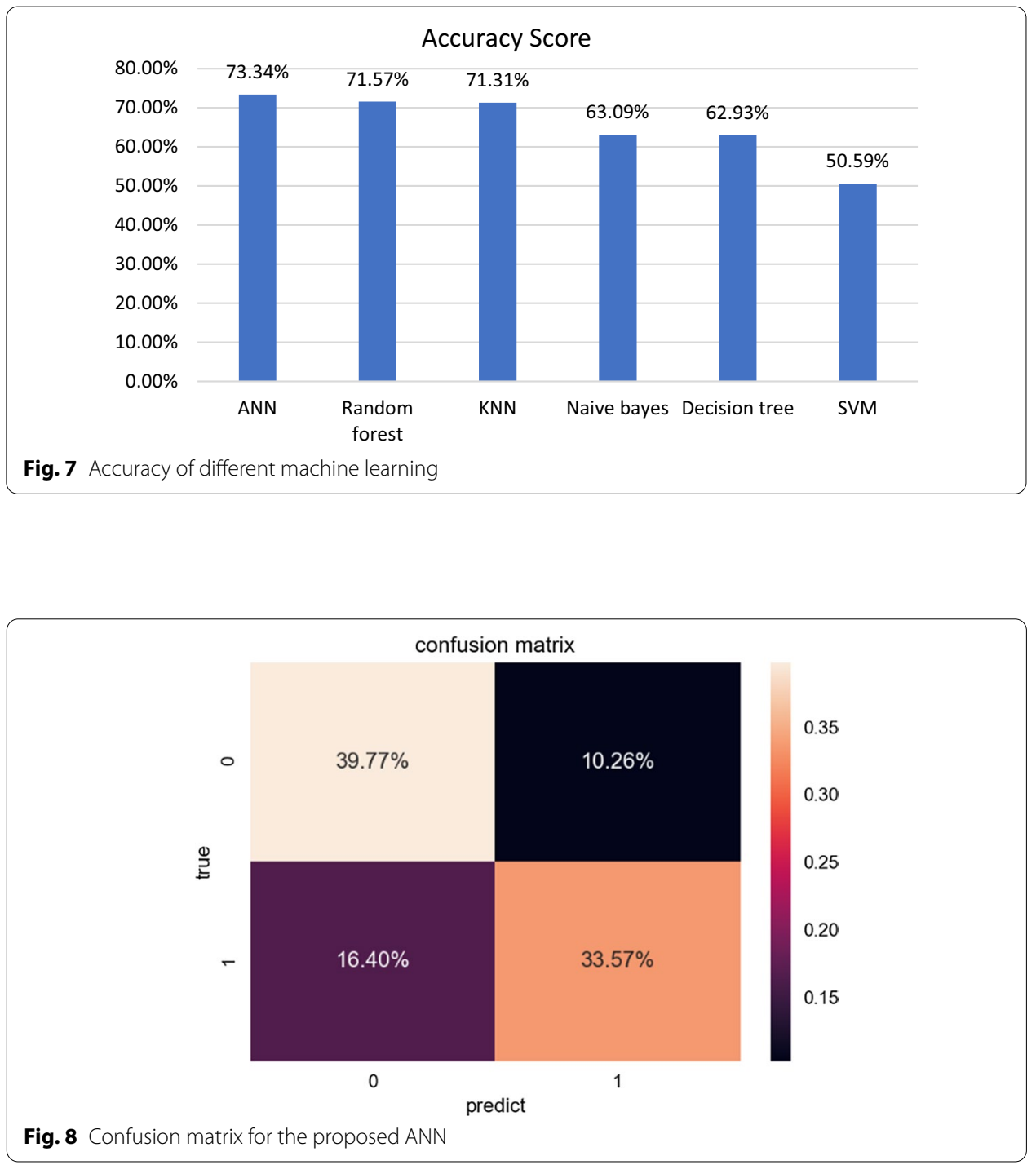

\subsection{Analysis for anomaly detection}

As shown in Fig. 7, the accuracy of the proposed shallow neural network is $73.34 \%$, which is higher than other machine learning algorithms. The proposed shallow neural network has only one hidden layer with 16 neurons, so the computational complexity is very low, and real-time monitoring of multiple users can be achieved.

In the anomaly detection at the MEC layer, we pay more attention to whether an anomaly can be detected, rather than paying special attention to detecting normal as an anomaly. Because even if a healthy person is misdiagnosed as a patient with cardiovascular disease, his life safety will not be threatened. However, if a patient attacked by cardiovascular disease is misdiagnosed as a healthy person, and thus misses an effective treatment method, it will greatly threaten his life safety. Therefore, in the confusion matrix, we are more concerned about false negatives (FN) than true positives (TP), false positives (FP), and true negatives (TN). As shown in Fig. 8, the FN accounted for $16.40 \%$ of the entire test set. 


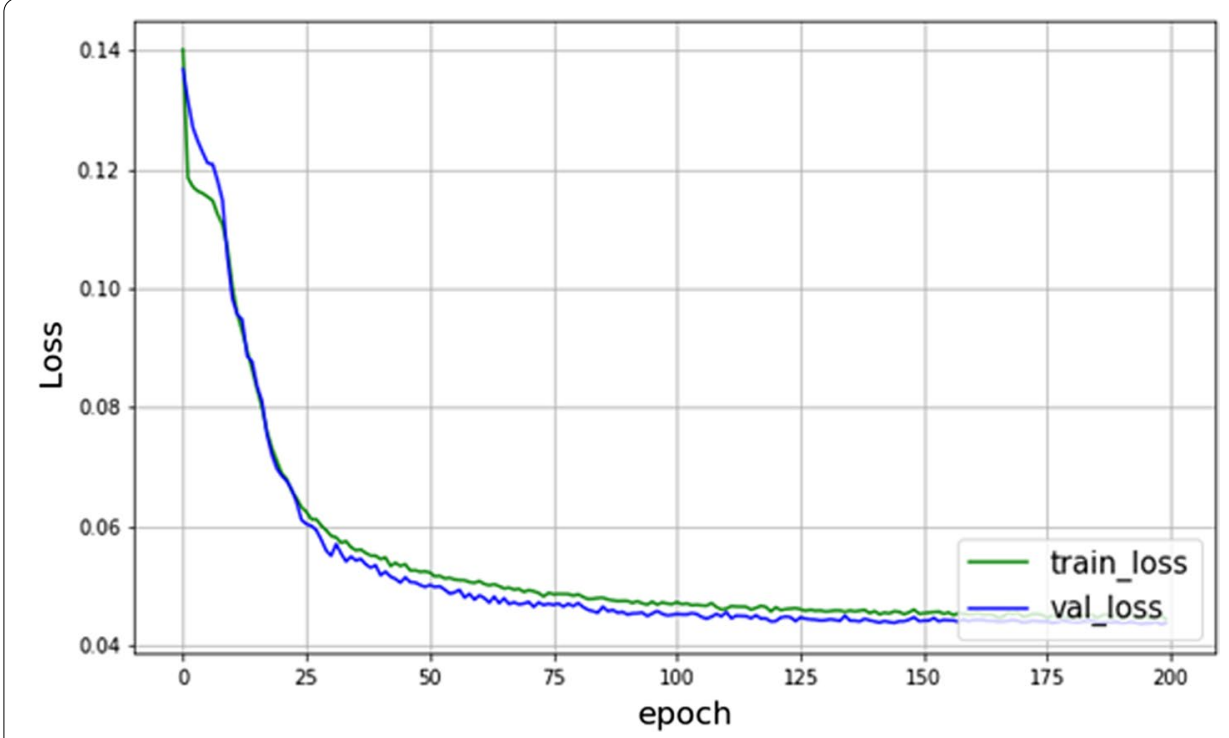

Fig. 9 The loss function curve of D-CNN model

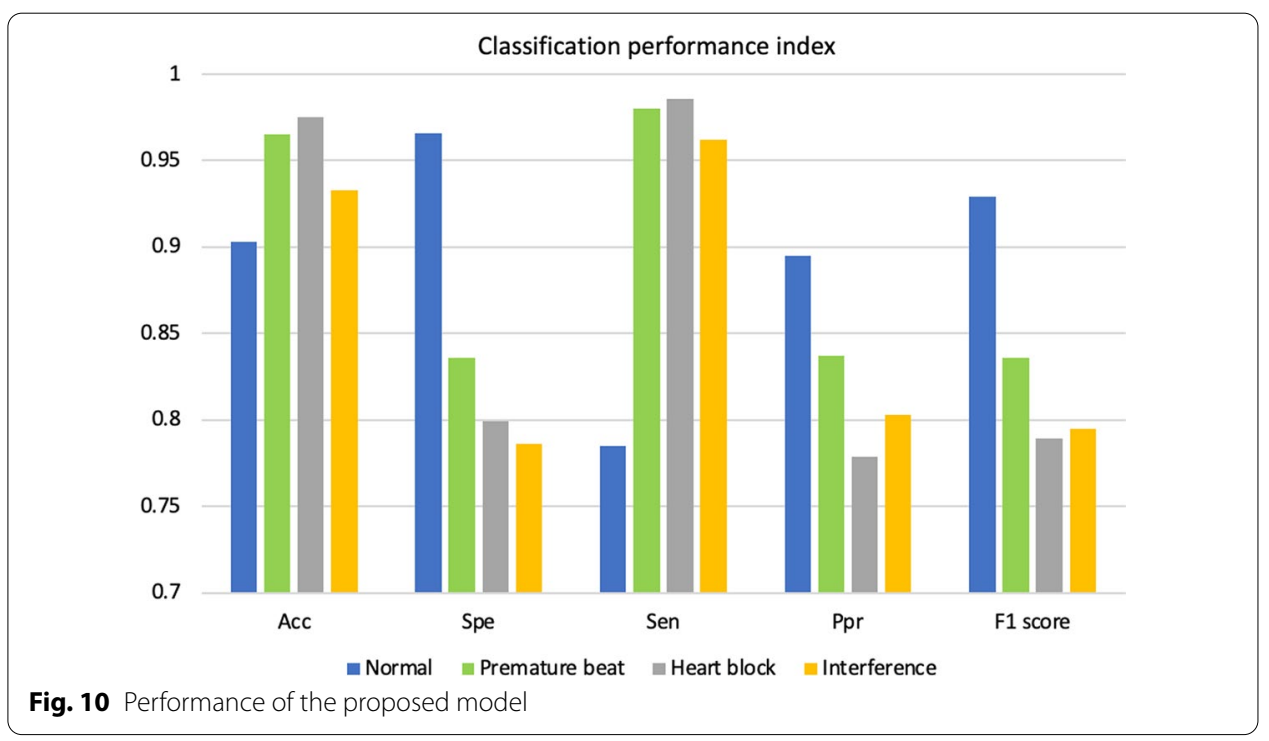

\subsection{Analysis for in-depth detection}

The loss function curve of proposed 1D-CNN model is shown in Fig. 9. Obviously, the loss function shows a downward trend with the increase in the epoch, and eventually remains basically unchanged. The cross-validation loss and training loss are basically the same as the epoch increases, which shows that our model has good generalization ability.

Figure 10 shows the performance of the four categories predicted by the model. These results show that the model is calculated through the test set. We selected the following five indicators: Acc (Accuracy), Spe (Specificity), Sen (Sensitivity), Ppr (Positive predictive rate) and F1 score as the main indicators to measure the performance of the model. Among the four categories, the model performs best in normal 
category. All the five indexes can reach above 0.9 , which proves that the positive prediction accuracy of the model is very high. The model also has high accuracy in distinguishing negative and positive samples in other three categories. One of the important reasons is that negative samples account for the majority of the samples in these three categories. Therefore, more negative sample decision features are learned by the model. In this study, the number of cardiac block categories recorded was the least in the ECG data set used. During the training process, the model tends to judge the type of heart block with negative samples, because the ratio of samples with heart block (positive samples) to samples without heart block (negative samples) is about 1:20, which also leads to the relatively low scores of SPE and PPR. Similarly, higher Acc values show that the classification accuracy of the model is very high. In the ECG dataset we use, there are fewer records with disease than those without disease. Therefore, the high performance of the model in classification accuracy mainly comes from health records. However, in specific medical scenarios, the prediction of disease records is very important.

\section{Conclusions}

We propose a smart an ambient backscatter communication based smart 5G IoT network. The system consists of two parts, namely a real-time data transmission system based on ambient backscatter communication and a real-time big data analysis system based on the combination of a shallow neural network and a deep neural network. A real-time data transmission system based on ambient backscatter communication can extend the standby time of health data collection equipment, reduce the size of the equipment, and increase the comfort of wearing. The real-time big data analysis system combining the shallow neural network and the deep neural network can greatly reduce the pressure caused by the frequent deep neural network calculations of the MEC, and greatly reduce the energy consumed by the MEC for remote real-time monitoring.

\footnotetext{
Abbreviations

IoT: Internet of Things; MEC: Mobile Edge Computing; RF: Radio Frequency; CR: Cooperative Receiver; BD: Backscatter Device; PT: Primary Transmitter; PR: Primary Receiver; ANN: Artificial Neural Network; CNN: Convolutional Neural Network; ECG: Electrocardiogram; SBP: Systolic Blood Pressure; DBP: Diastolic Blood Pressure; TP: True Positives; FN: False Negatives; FP: False Positives; TN: True Negatives; Acc: Accuracy; Spe: Specificity; Sen: Sensitivity; Ppr: Positive predictive rate.
}

\section{Acknowledgements}

Not applicable.

\section{Authors' contributions}

In this paper, QL conceived, designed and wrote the study. SS guided the structure and research content of this paper. $Y Z$ and $X Y$ provided reliable suggestions when revising the manuscript. $Y Z$ gave suggestions on the role of active communication and passive communication in real-time data transmission systems. XY gave suggestions on the role of shallow neural networks and deep neural networks in real-time big data analysis systems, and corrected grammatical errors in the full paper. All authors read and approved the final manuscript.

\section{Funding}

This work was supported in part by the National Natural Science Foundation of China under Project 61471066, and in part by the Open Project Fund of the National Key Laboratory of Electromagnetic Environment, China, under Grant 201600017.

\section{Availability of data and materials}

The authors keep the analysis and simulation data set. Part of the data set is kept confidential and not public, but some public data can be obtained from the authors according to reasonable requirements.

\section{Competing interests}

The authors declare that they have no competing interests. 


\begin{abstract}
Author details
${ }^{1}$ School of Information and Communication Engineering, Beijing University of Posts and Telecommunications, Beijing 100876, China. ${ }^{2}$ State Key Laboratory of Information Photonics and Optical Communications, Beijing University of Posts and Telecommunications, Beijing 100876, China.
\end{abstract}

Received: 10 September 2020 Accepted: 1 February 2021

Published online: 17 February 2021

\title{
References
}

1. N. Javaid, A. Sher, H. Nasir, N. Guizani, Intelligence in loT-based 5G networks: opportunities and challenges. IEEE Commun. Mag. 56(10), 94-100 (2018)

2. L. Guo, F. Chen, L. Chen, X. Tang, 'The building of cloud computing environment for e-health', in: Proceedings of the IEEE International Conference on E-Health Networking, Digital Ecosystems and Technologies, (2010), pp. 89-92.

3. S. Lee, S. Lee, M. Shin, Low cost MEC server placement and association in $5 G$ networks, in: 2019 International Conference on Information and Communication Technology Convergence (ICTC), Jeju Island, Korea (South), (2019), pp. 879-882.

4. K. He, X. Zhang, S. Ren, J. Sun, Deep residual learning for image recognition, in: CVPR (2016).

5. G. Yang et al., A health-loT platform based on the integration of intel- ligent packaging, unobtrusive bio-sensor, and Intelligent medicine box. IEEE Trans. Ind. Inf. 10(4), 2180-2191 (2014)

6. U. K. Prodhan, M. Z. Rahman, I. Jahan, A. Abid, M. Bellah, Development of a portable telemedicine tool for remote diagnosis of telemedicine application, in: 2017 International Conference on Computing, Communication and Automation (ICCCA), Greater Noida (2017), pp. 287-292.

7. M.R. Yuce, T. Dissanayake, Easy-to-swallow wireless telemetry. IEEE Microw. Mag. 13(6), 90-101 (2012)

8. X. Chen, X. Zhang, L. Zhang, X. Li, N. Qi, H. Jiang, Z. Wang, A wireless capsule endoscope system with low-power controlling and processing ASIC. IEEE Trans. Biomed. Circuits Syst. 3(1), 11-22 (2009)

9. N. Van Huynh, D.T. Hoang, X. Lu, D. Niyato, P. Wang, D.I. Kim, Ambient backscatter communications: a contemporary survey. IEEE Commun. Surv. Tutor. 20(4), 2889-2922 (2018)

10. V. Liu, A. Parks, V. Talla, S. Gollakota, D. Wetherall, J.R. Smith, Ambient backscatter: wireless communication out of thin air, in: Proceedings of ACM SIGCOMM, Hong Kong, (2013), pp. 39-50

11. X. Lu, D. Niyato, H. Jiang, D.I. Kim, Y. Xiao, Z. Han, Ambient backscatter assisted wireless powered communications. IEEE Wirel. Commun. 25(2), 170-177 (2018)

12. F. Jameel, R. Duan, Z. Chang, A. Liljemark, T. Ristaniemi, R. Jantti, Applications of backscatter communications for healthcare networks. arXiv 2019, arXiv:1906.09209.

13. S. Sun, M. Kadoch, L. Gong, B. Rong, Integrating network function virtualization with SDR and SDN for $4 G / 5 G$ networks. IEEE Netw. 29(3), 54-59 (2015)

14. N. Zhang, N. Cheng, A.T. Gamage, K. Zhang, J.W. Mark, X. Shen, Cloud assisted HetNets toward 5 G wireless networks. IEEE Commun. Mag. 53(6), 59-65 (2015)

15. Y. Wu, B. Rong, K. Salehian, G. Gagnon, Cloud transmission: a new spectrum-reuse friendly digital terrestrial broadcasting transmission system. IEEE Trans. Broadcast. 58(3), 329-337 (2012)

16. B. Rong, Y. Qian, K. Lu, H. Chen, M. Guizani, Call admission control optimization in WiMAX networks. IEEE Trans. Veh. Technol. 57(4), 2509-2522 (2008)

17. N. Chen, B. Rong, X. Zhang, M. Kadoch, Scalable and flexible massive MIMO precoding for $5 \mathrm{G}$ H-CRAN. IEEE Wirel. Commun. 24(1), 46-52 (2017)

18. A.Y. Hannun, P. Rajpurkar, M. Haghpanahi et al., Cardiologist-level arrhythmia detection and classification in ambulatory electrocardiograms using a deep neural network. Nat. Med. 25(1), 65-69 (2019)

19. G. Yang, Q. Zhang, Y.-C. Liang, Cooperative ambient backscatter communications for green Internet-of-Things. IEEE Internet Things J. 5(2), 1116-1130 (2018)

\section{Publisher's Note}

Springer Nature remains neutral with regard to jurisdictional claims in published maps and institutional affiliations. 